\title{
The chemical evolution of manganese in different stellar systems
}

\author{
G. Cescutti ${ }^{1}$, F. Matteucci ${ }^{1,2}$, G. A. Lanfranchi ${ }^{3}$, and A. McWilliam ${ }^{4}$
}

\author{
1 Dipartimento di Astronomia, Universitá di Trieste, via G.B. Tiepolo 11, 34131, Italy \\ e-mail: cescutti@oats.inaf.it \\ 2 INAF Osservatorio Astronomico di Trieste, via G.B. Tiepolo 11, 34131, Italy \\ 3 Núcleo de Astrofísica Teórica, Universidade Cruzeiro do Sul, R. Galvão Bueno 868, Liberdade, 01506-000, São Paulo, SP, Brazil \\ ${ }^{4}$ Observatories of the Carnegie Institution of Washington, 813 Santa Barbara St., Pasadena, CA, USA
}

Received 7 July 2008 / Accepted 28 August 2008

\begin{abstract}
Aims. We model the chemical evolution of manganese relative to iron in three different stellar systems: the Solar neighbourhood, the Galactic bulge, and the Sagittarius dwarf spheroidal galaxy, and we compare our results with recent and homogeneous observational data sets.

Methods. We adopt three chemical evolution models able to reproduce the main properties of the Solar vicinity, the Galactic bulge, and the Sagittarius dwarf spheroidal. We then compare different stellar yields in order to identify the most appropriate set to match the observational data in these systems.

Results. We compute the evolution of manganese in the three systems and find that to reproduce simultaneously the measurements of $[\mathrm{Mn} / \mathrm{Fe}]$ versus $[\mathrm{Fe} / \mathrm{H}]$ in the Galactic bulge, the Solar neighbourhood and Sagittarius, the type Ia supernova (SN) Mn yield must be metallicity-dependent.

Conclusions. We conclude that modelling different histories of star formation in the three systems are insufficient to reproduce the different behaviour of the $[\mathrm{Mn} / \mathrm{Fe}]$ ratio, unlike the situation for $[\alpha / \mathrm{Fe}]$; rather, it is necessary to invoke metallicity-dependent type Ia SN Mn yields, as originally suggested by McWilliam, Rich \& Smecker-Hane.
\end{abstract}

Key words. nuclear reactions, nucleosynthesis, abundances - Galaxy: abundances - Galaxy: evolution

\section{Introduction}

McWilliam et al. (2003) considered the abundance trend of Mn in three contrasting stellar populations: a sample of Galactic bulge K giants, stars in the Solar neighbourhood, and stars in the dwarf spheroidal galaxy Sagittarius (Sgr dSph). They found that the trend of $[\mathrm{Mn} / \mathrm{Fe}]$ in the Galactic bulge followed more or less the relation of the Solar vicinity, but most stars of Sgr dSph were found to be $[\mathrm{Mn} / \mathrm{Fe}]$ deficient by $\sim 0.2 \mathrm{dex}$. To explain this result, they concluded that one has to assume that the yields of Mn from both type II and type Ia SNe should depend on the initial stellar metallicity. This conclusion was at variance with a suggestion by Gratton (1989) that $\mathrm{Mn}$ is overproduced in type Ia SNe, relative to type II SNe. Gratton (1989) observed that [Mn/Fe] versus $[\mathrm{Fe} / \mathrm{H}]$ in Solar vicinity stars have the opposite behaviour to the $[\alpha / \mathrm{Fe}]$ ratios: $[\mathrm{Mn} / \mathrm{Fe}] \sim-0.4$ dex and constant for stars with $[\mathrm{Fe} / \mathrm{H}]<-1.0 \mathrm{dex}$, and increases to solar values for stars with $[\mathrm{Fe} / \mathrm{H}]>-1.0$ dex. As is well known, first Tinsley (1979) and then Greggio \& Renzini (1983) and Matteucci \& Greggio (1986) explained the trend of the $\alpha$-elements (e.g. O, Mg, Si, Ca, Ti), relative to $\mathrm{Fe}$, as being due to the different roles played by type Ia and II SNe in Galactic chemical enrichment. In particular, the higher than solar $[\alpha / \mathrm{Fe}]$ ratio in halo stars is interpreted as being due to the pollution of type II SNe, which explode on very short timescales (of the order of Myr to tenths of Myr) and produce roughly constant $[\alpha / \mathrm{Fe}]$ ratios. The $[\alpha / \mathrm{Fe}]$ ratio then decreases to the Solar value in disk stars because the bulk of Fe is produced by type Ia $\mathrm{SNe}$ on timescales ranging from 30-40 Myr to 10 Gyr. This interpretation is known as the "time-delay model" and represents the most likely interpretation of alpha-element abundance ratios in galaxies so far. Type II SNe originate from the core-collapse of massive stars $\left(M>10 M_{\odot}\right)$, whereas it is believed that type Ia $\mathrm{SNe}$ are produced by mass-accretion in binary $\mathrm{C}-\mathrm{O}$ white dwarfs systems, and the subsequent explosion when the white dwarf mass reaches the Chandrasekhar limit. Two main scenarios can lead to this situation: i) the single degenerate, where a white dwarf explodes after accreting material from a red giant or a main-sequence companion (Whelan \& Iben 1973); ii) the double degenerate, where the explosion follows the merger of two C-O white dwarfs (Iben \& Tutukov 1984), due to loss of angular momentum from gravitational wave emission. There is no consensus as yet on which scenario is more appropriate or if both operate in galaxies. In both scenarios, type Ia $\mathrm{SNe}$ begin to explode after 30-40 Myr, and this continues for a Hubble time. The maximum type Ia SN rate depends not only on the assumed progenitor model but also on the assumed star formation history, which is short in lenght for systems with strong bursts of star formation (e.g. 0.5 Gyr in the Galactic bulge), $1.0-1.5$ Gyr in the Solar vicinity and several Gyrs in slow starforming systems, such as irregular galaxies (Matteucci \& Recchi 2001). Therefore, the bulk of Fe is always produced with a delay relative to the $\alpha$-elements produced by type II SNe. Manganese is an iron-peak element produced in both type II and Ia SNe. Nucleosynthesis calculations for massive stars by Arnett (1971) and Woosley \& Weaver (1995, hereafter WW95), among others, found that yields of $\mathrm{Mn}$ increased with increasing metallicity (in fact with increasing neutron excess). Metallicity-dependent 
yields for type Ia SN have been largely unexplored; Ohkubo et al. (2006) demonstrated that the yields of both $\mathrm{Ni}$ and $\mathrm{Mn}$ increase with metallicity in type Ia SNe, although this conclusion was sensitive to the details of the adopted hydrodynamical models.

In this paper, we adopt three detailed chemical evolution models for the Galactic bulge, Solar vicinity and Sgr dSph. In all models, we adopt the same yields but different star formation histories that are tuned to reproduce the majority of the observational constraints for the three systems. To explain the $[\mathrm{Mn} / \mathrm{Fe}]$ simultaneously in the three systems, we show that the time-delay model alone is insufficient and we must assume that the yields of $\mathrm{Mn}$ from type Ia SNe are metallicity-dependent. The paper is organized as follows: in Sect. 2, we briefly describe the observational data; in Sect. 3, we describe the chemical evolution models. In Sect. 4, the nucleosynthesis prescriptions are given. In Sect. 5, we discuss our results, and in Sect. 6, we draw our conclusions.

\section{Observational data}

The observational data used in this paper for the Galactic bulge were taken from McWilliam et al. (2003). For Sgr dSph, the chemical abundance ratios of 14 stars were from McWilliam et al. (2003); from the work of Sbordone et al. (2007), we derived data on the chemical composition of 12 stars in the Sgr $\mathrm{dSph}$ main body and 5 more stars in the associated globular cluster Tarzan 7. The data for the solar neighbourhood shown in this paper are the abundances reported by Reddy et al. (2003) and Reddy et al. (2006); they are not the only data available, were selected to represent a homogeneous sample.

\section{The chemical evolution models}

For studying the chemical evolution of the solar neighbourhood, we adopted the model of François et al. (2004), which is an revised version of an original model by Chiappini et al. (1997). This model assumes that the Galaxy formed by means of two main accretion episodes, one giving rise to the halo and thick disk and the other forming the thin disk. The infalling gas is always assumed to be of primordial composition. Detailed nucleosynthesis from low and intermediate mass stars, and type Ia and type II SNe are taken into account. The IMF is taken from Scalo (1986). Further details of this model were provided in François et al. (2004).

For the Galactic bulge, we adopted the model of Ballero et al. (2007), which assumed a rapid formation, of timescale 0.3-0.5 Gyr, from gas accumulated during halo collapse. The efficiency of star formation (star formation per unit mass of gas) is 20 times higher (i.e. $20 \mathrm{Gyr}^{-1}$ ) than in the Solar vicinity $\left(1 \mathrm{Gyr}^{-1}\right)$. The IMF is flatter than in the Solar vicinity, as required by the observed bulge stellar metallicity distribution. Ballero et al. (2007) described this model in more detail.

To study Sgr dSph, we have adopted a model developed specifically for this galaxy by Lanfranchi et al. (2004, 2006). This model assumes that Sgr dSph formed stars over several Gyrs, as suggested by its color-magnitude diagram, and that a strong galactic wind, triggered by supernovae, was responsible for its gas loss. While the star formation efficiency is similar to that of the Solar vicinity, the effect of significant gas loss is similar to that of a lower star formation efficiency. In particular, we assume that the star formation rate is given by a simple Schmidt law:

$\psi(t)=v \sigma_{\mathrm{gas}}$

where $\psi(t)$ is the star formation rate, $v=1 \mathrm{Gyr}^{-1}$ is the star formation efficiency (namely the star formation rate per unit mass of gas) and $\sigma_{\text {gas }}$ is the surface gas density. The wind rate is then assumed to be:

$W(t)=\lambda \psi(t)$

with $\lambda$ being a parameter whose most appropriate value for Sgr $\mathrm{dSph}$ is $\lambda=13.0$. The assumed IMF is also flatter than in the Solar vicinity (Salpeter 1955). The necessity of varying the IMF between the three models is dictated by the stellar metallicity distributions in the three stellar systems: for both the bulge and $\mathrm{Sgr} \mathrm{dSph}$ an IMF that is flatter than the disk IMF is required.

According to the time delay model, the predicted $[\alpha / \mathrm{Fe}]$ ratios behave differently in the three systems because of differences in the star formation histories (see Matteucci 2003), in the sense that we expect a long plateau with supersolar $[\alpha / \mathrm{Fe}]$ ratios in the bulge because of its rapid star formation. Type II $\mathrm{SNe}$ in fact produce a significant amount of Fe before the bulk of Fe is formed by type Ia SNe. The opposite occurs for slow, star-forming systems, such as dwarf spheroidals. The supersolar $[\alpha / \mathrm{Fe}]$ plateau is then far shorter in duration than in the Solar vicinity since when the type Ia SNe produce the bulk of Fe, the $[\mathrm{Fe} / \mathrm{H}]$ in the interstellar medium is quite low. This produces a situation where we expect low $[\alpha / \mathrm{Fe}]$ ratios at low metallicities as opposed to the high $[\alpha / \mathrm{Fe}]$ ratios at high metallicities expected for the bulge. The observed behaviour of the $[\alpha / \mathrm{Fe}]$ ratios in these three systems is in good agreement with these predictions (e.g. François et al. 2004; Ballero et al. 2007; Lanfranchi et al. 2006).

In the following sections, we test whether a time-delay scenario, where type Ia SNe produce the bulk of the Mn (as suggested Gratton 1989) can predict the observed [Mn/Fe] ratios in these systems.

\section{Nucleosynthesis prescriptions}

As already mentioned, $\mathrm{Fe}$ and $\mathrm{Mn}$ are produced by type Ia and II SNe. In our chemical evolution calculations, we explore the consequences of three nucleosynthesis prescriptions: (1.) fixed, metallicity-independent, $\mathrm{Mn}$ and $\mathrm{Fe}$ yields for type Ia and II SNe; (2.) metallicity-dependent type II SNe yields with fixed, metal-independent, yields for type Ia SNe; and (3.) metaldependent Mn yields for both type Ia and type II SNe.

In our first model, we utilise the metal-independent yields from François et al. (2004). For type II SNe, they found that the WW95 results provide the closest description to Solar vicinity data; in fact, no modifications were required for the yields of Fe computed for Solar chemical composition. However, in order to fit the observed $[\mathrm{Mn} / \mathrm{Fe}]$ trend François et al. (2004) modified the WW95 Mn yields for type II SNe, with a $\sim 70 \%$ increase for 13-18 $M_{\odot}$ progenitors and a $\sim 60 \%$ decrease for $30-$ $40 M_{\odot}$ progenitors. For type Ia SNe, François et al. (2004) employed the, unmodified, metallicity-independent Iwamoto et al. (1999) yields. Effectively, the François et al. (2004) used the agemetallicity relation and modified the Mn yields as a function of supernova progenitor mass to reproduce the observed trend of $[\mathrm{Mn} / \mathrm{Fe}]$ with metallicity in the Solar vicinity. These yields are therefore fine-tuned to the formation timescale of the thin disk. 


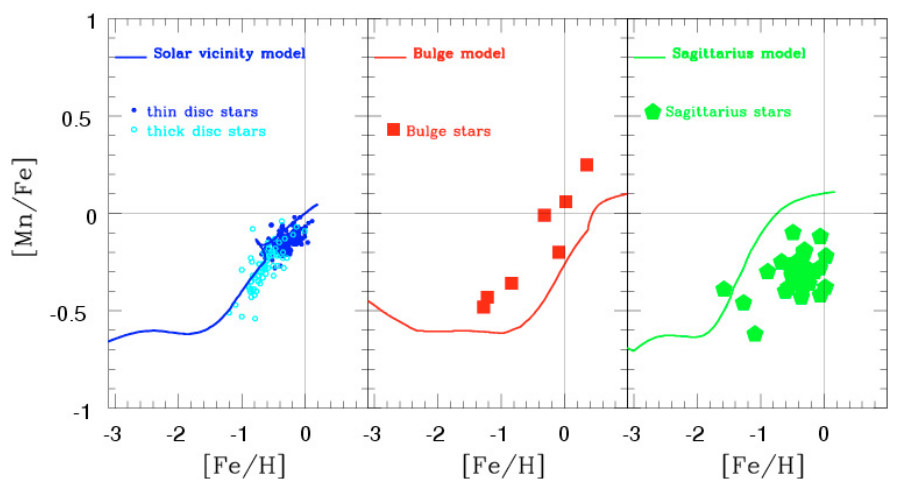

Fig. 1. Results with François et al. (2004) set of yields. We present the $[\mathrm{Mn} / \mathrm{Fe}]$ versus $[\mathrm{Fe} / \mathrm{H}]$ ratios in the three different stellar systems, from left to right: Solar neighbourhood, Galactic bulge and Sagittarius Dwarf Spheroidal. For the data, see Sect. 2.

We employed these yields for our initial chemical evolution calculations to predict the $[\mathrm{Mn} / \mathrm{Fe}]$ trend for the metal-independent time-delay scenario.

For our second nucleosynthesis prescription, we chose to use the metallicity-dependent Mn and Fe yields of WW95 for type II SNe, and we adopted the metallicity-independent theoretical yields by Iwamoto et al. (1999) for the yields of type Ia SNe.

Finally, for the third nucleosynthesis recipe we again employed the WW95 metal-dependent yields for type II SNe but we also used metal-dependent Mn yields for type Ia SNe. For the metal-dependent type Ia SN Mn yields, we modified the values of Iwamoto et al. (1999) by introducing the following metallicity dependence:

$Y_{\mathrm{Mn}}(z)=Y_{\mathrm{Mn}}^{\mathrm{Iwamoto}}\left(\frac{z}{z_{\odot}}\right)^{0.65}$

where $Z$ is the global metal content of the type Ia SN systems at birth. The form of the metal-dependent yields was obtained by requiring that the model predictions fit the observational data. Badenes et al. (2008) found that the $\mathrm{Mn} / \mathrm{Cr}$ ratio in the ejecta of type Ia SNe depends on $Z$, and their modelled metallicitydependent Mn yield was similar to that in Eq. (3), which supports our choice. Nucleosynthesis calculations by Ohkubo et al. (2006) also suggested a dependence of the yields of both Ni and $\mathrm{Mn}$ on metallicity in type Ia SNe.

\section{Results}

\subsection{Results with François et al. yields}

In Fig. 1, we show the results obtained with the yields of François et al. (2004), and as expected, the data relative to the stars of the Solar vicinity are reproduced well. Here and in the following figures, the results of all models are normalized by the predicted solar abundances, namely the abundances predicted for the interstellar medium in the Solar vicinity $4.5 \mathrm{Gyr}$ ago. We note that our predicted solar values for most of the considered abundances and, in particular for those studied in this paper, differ from the Asplund et al. (2005) solar values by a maximum of $\sim 0.06 \mathrm{dex}$.

On the other hand, Fig. 1 shows an unsatisfactory comparison between the time delay model predictions and the observed bulge $[\mathrm{Mn} / \mathrm{Fe}]$ trend with $[\mathrm{Fe} / \mathrm{H}]$ : there is an offset between the

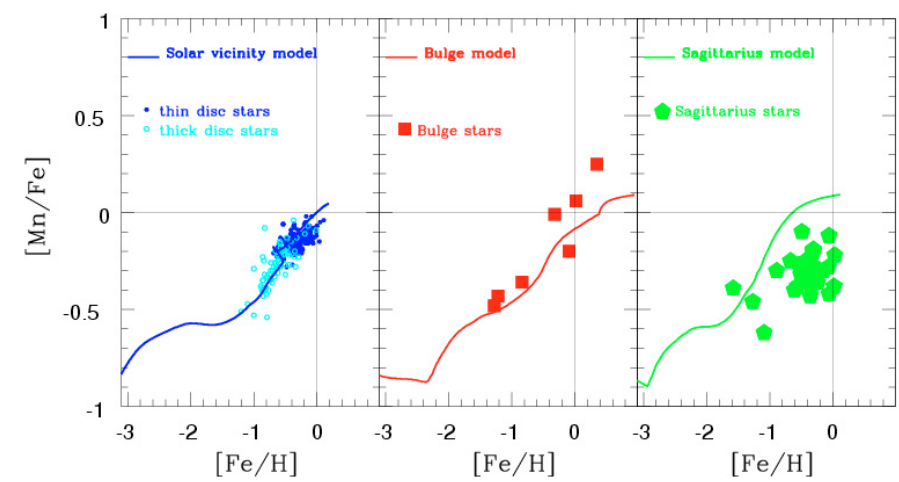

Fig. 2. Results with the WW95 metallicity-dependent type II SN yields, with constant, Iwamoto et al. (1999) type Ia SN Mn yields. The [Mn/Fe] versus $[\mathrm{Fe} / \mathrm{H}]$ ratios in the three different stellar systems, from left to right: Solar neighbourhood, bulge and Sagittarius Dwarf Spheroidal galaxy. For the data, see Sect. 2.

data and the results of the bulge model, which predicts lower values of the $[\mathrm{Mn} / \mathrm{Fe}]$ ratio for all metallicities. Under the constraint of metallicity-independent Mn yields, the time-delay model for the Solar neighbourhood requires that the bulk of the $\mathrm{Mn}$ is produced by type Ia SNe. Because of the rapid formation of the bulge (suggested by its alpha-element abundances), the contribution of type Ia SNe to the bulge composition is smaller than for the solar vicinity; the model therefore predicts in this paradigm less $\mathrm{Mn}$ production in the bulge, and therefore lower $[\mathrm{Mn} / \mathrm{Fe}]$ ratios (in contrast to the observations).

With this set of yields, the results of Sgr dSph model also do not match the observed $[\mathrm{Mn} / \mathrm{Fe}]$ trend with $[\mathrm{Fe} / \mathrm{H}]$ : the theoretical curve passes through the observed $[\mathrm{Mn} / \mathrm{Fe}]$ values only at $[\mathrm{Fe} / \mathrm{H}] \sim-1.5 \mathrm{dex}$, but for higher metallicities the predicted trend is too high compared with the data. This can be understood as a result of the high frequency of type Ia $\mathrm{SNe}$ in the the Sgr dSph model (suggested by the low alpha/Fe ratios), which produce more Mn for metallicity-independent Mn yields in the time-delay scenario. The prediction of this paradigm is therefore for enhanced $[\mathrm{Mn} / \mathrm{Fe}]$ ratios in the $\mathrm{Sgr} \mathrm{dSph}$ (again in contradiction to the observations).

\subsection{Results with Woosley \& Weaver yields}

To improve the match between our models and the data, in particular for the bulge and Sgr dSph, we now explore the use of the metallicity-dependent WW95 yields from type II SNe, but with the constant (i.e. metallicity-independent) $\mathrm{Mn}$ and $\mathrm{Fe}$ yields for type Ia SNe from Iwamoto et al. (1999); the results are shown in Fig. 2.

The results of the Solar neighbourhood model still agree with the observational data.

The model results for the bulge are also more promising. Up to solar $[\mathrm{Fe} / \mathrm{H}]$, the predictions reproduce the observational data well. However, for supersolar metallicities the results of the model tend to underestimate slightly the value of $[\mathrm{Mn} / \mathrm{Fe}]$ in the bulge stars. Because of the high star formation rate in the bulge, type Ia SNe finally occur at late times (and high $[\mathrm{Fe} / \mathrm{H}]$ ). The $\mathrm{Mn}$ deficit at supersolar metallicities in this bulge model might therefore reasonably be due to a problem with $\mathrm{Mn}$ from type Ia SNe.

The results from this model for the Sgr dSph are still in poor agreement with the data. The model shows a strong increase in the $[\mathrm{Mn} / \mathrm{Fe}]$ ratio at $[\mathrm{Fe} / \mathrm{H}] \sim-1.5 \mathrm{dex}$, at variance with the 


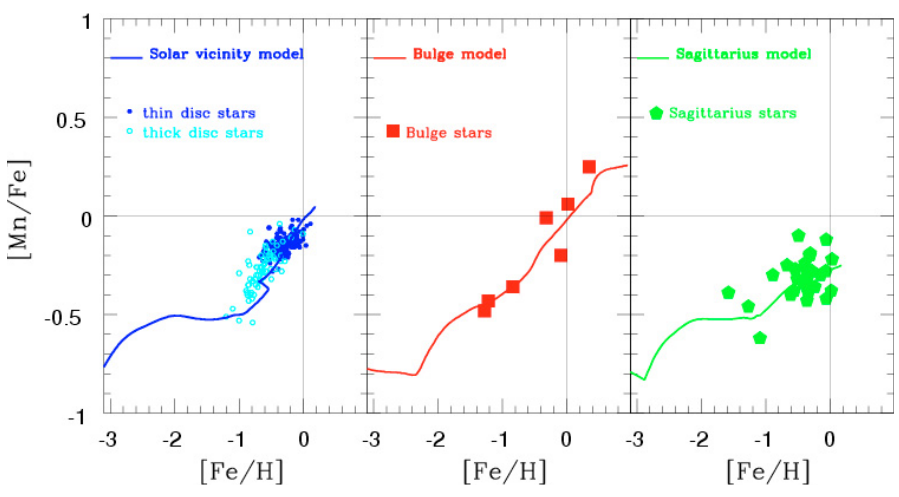

Fig. 3. Results with metallicity dependence in both type II SNe and type Ia SN yields for $\mathrm{Mn}$. The $[\mathrm{Mn} / \mathrm{Fe}]$ vs $[\mathrm{Fe} / \mathrm{H}]$ ratios in three different stellar systems, from left to right: Solar neighbourhood, bulge and Sagittarius Dwarf Spheroidal. For the data, see Sect. 2.

data. Given the prominent role of type Ia SNe in Sgr dSph, this $\mathrm{Mn}$ overprediction is probably related to the type Ia SN Mn yields.

\subsection{Results with Woosley \& Weaver yields and metallicity dependent Mn yields for type la SNe}

In the last set of chemical evolution models tested, both the type Ia and II SN yields of Mn are dependent on the metallicity. In particular, the Mn yields from type Ia SNe follow Eq. (3). The yields are constrained to maintain agreement between the chemical evolution model predictions for $[\mathrm{Mn} / \mathrm{Fe}]$ versus $[\mathrm{Fe} / \mathrm{H}]$, and the Solar vicinity observational data.

At the same time, the supersolar data in the bulge are described well. The use of this new set of yields therefore improve the predictions of the bulge model, presumably because the Mn contribution from late, high-metallicity, type Ia $\mathrm{SNe}$ are modelled more correctly.

Finally, the most interesting result is the excellent agreement between the Sgr dSph model and the data. This is due to the reduced contributions to the Mn enrichment by low-metallicity type Ia SNe, compared with the time-delay metallicity-independent model.

A very important conclusion is that the yield of Mn produced by type Ia SN must be metallicity-dependent to be able to reproduce the Sgr dSph abundance data. We also obtain excellent agreement also for the bulge and Solar vicinity stars. We emphasize that it would not have been possible to obtain this result without the simultaneous comparison of the three independent stellar systems, which are characterized by different starformation timescales. This hypothesis, that the type Ia SN Mn yield must be dependent on metallicity, was first suggested by McWilliam et al. (2003).

\section{Conclusions}

By fitting simultaneously the $[\mathrm{Mn} / \mathrm{Fe}]$ trend in the three independent stellar systems of the Galactic bulge, the solar vicinity, and the Sgr dSph galaxy, we have shown quantitatively, for the first time and by means of galactic chemical evolution models, that the type Ia SN Mn yields must depend on metallicity.

We have adopted detailed chemical evolution models, chosen to reproduce the main chemical characteristics of the three stellar systems, using identical element yields but different star formation histories. We have shown clearly that the "timedelay model" alone cannot explain the different behaviour of the $[\mathrm{Mn} / \mathrm{Fe}]$ in the Galactic bulge, Solar vicinity, and Sgr dSph galaxy, as it can explain $[\alpha / \mathrm{Fe}]$ ratios. In the case of the $[\alpha / \mathrm{Fe}]$ ratios, the same models can reproduce the observed $[\alpha / \mathrm{Fe}]$ versus $[\mathrm{Fe} / \mathrm{H}]$ trends in the three stellar systems well without the need to invoke metallicity-dependent yields. This is because the production of $\alpha$ and Fe elements is only mildly dependent on the initial stellar metallicity. We propose a functional form of the metallicity-dependent Mn yields in type Ia SNe $\left(\propto Z^{0.65}\right)$, which should be tested by nucleosynthesis calculations. Our result is supported by Ohkubo et al. (2006), who suggested that the yields of $\mathrm{Mn}$ from type Ia SNe have a strong dependence on metallicity, and by Badenes et al. (2008), who demonstrated that the $\mathrm{Mn}$ to $\mathrm{Cr}$ ratio in the ejecta of type Ia SNe depend on the metallicity of the progenitor star in a way similar to that we have suggested.

It is worth noting that in this work we have taken the Reddy et al. (2003, 2006) abundances for the Solar vicinity stars at face value. However, we note that McWilliam et al. (2003) found that a zero-point correction of +0.10 dex to the Reddy et al. (2003) $[\mathrm{Mn} / \mathrm{Fe}]$ values was required if the Sun is typical of stars in the range $-0.10 \leq[\mathrm{Fe} / \mathrm{H}] \leq+0.10$. We have re-investigated this issue and found that together the Reddy et al. (2003) and Reddy et al. (2006) data indicate a mean $[\mathrm{Mn} / \mathrm{Fe}]$ of solar $[\mathrm{Fe} / \mathrm{H}]$ stars at $-0.10 \pm 0.01 \mathrm{dex}$, with an rms scatter about the mean of 0.036 dex. Since the solar $[\mathrm{Mn} / \mathrm{Fe}]$ ratio is $2.77 \sigma$ above the mean for solar $[\mathrm{Fe} / \mathrm{H}]$ stars (which is equal to $99.7 \%$ above the mean in a Gaussian distribution), this zero-point shift is most likely due to systematic error in the Reddy et al. (2003, 2006) results, rather than a real Mn enhancement in the Sun. This is supported by the fact that other abundance studies require quite different zero-point corrections; for example the differential study, relative to the Sun, by Feltzing \& Gustafsson (1998) required a zero-point $[\mathrm{Mn} / \mathrm{Fe}]$ correction of $-0.06 \mathrm{dex}$. Unfortunately, we are unable to reproduce the measured $[\mathrm{Mn} / \mathrm{Fe}]$ trends as well if we apply a 0.10 dex zero-point shift to the Reddy et al. (2003, 2006) data. To do so would require a more complicated form of the metallicity-dependent $\mathrm{Mn}$ yields from type Ia SNe, instead of the simple power-law form adopted here. Alternatively, other complications might also be responsible, such as the type II SNe yields of WW95, or variations in the star formation and/or the mass-loss rates for Sgr dSph. Rather than explore a myriad of possibilities that may all provide a perfect fits to the Mn trends, we prefer to conclude with our main quantitative result, that the trend of $[\mathrm{Mn} / \mathrm{Fe}]$ with $[\mathrm{Fe} / \mathrm{H}]$ in the Galactic disk, the bulge, and the Sgr dSph can only be understood if the yield of Mn from type Ia SNe is metallicity-dependent.

Finally, we note that the low $[\mathrm{Mn} / \mathrm{Fe}]$ values measured in $\mathrm{Sgr}$ $\mathrm{dSph}$ stars close to solar metallicity is due, at least in part, to the lower metallicity of old Sgr dSph stars compared with those in the Solar neighborhood. The low average $[\mathrm{Fe} / \mathrm{H}]$ of the type Ia $\mathrm{SNe}$ in the Sgr dSph produces the lower [Mn/Fe] values, due to the metallicity-dependence of the $\mathrm{Mn}$ yield. The $[\mathrm{Mn} / \mathrm{Fe}]$ ratios of solar metallicity stars in dwarf galaxies is therefore linked to the mean metallicity of these galaxies. Since the metallicity distribution function of stars in dwarf galaxies is sensitive to the mass outflow and star-formation rates, the value of these parameters should be constrained by the observed $[\mathrm{Mn} / \mathrm{Fe}]$ ratios. We would therefore expect to observe similar trends for $[\mathrm{Mn} / \mathrm{Fe}]$ in the more massive dwarf galaxies (e.g. Large Magellanic Cloud), although less extreme than in lower mass dwarf galaxies. There should therefore be families of low-mass galaxies with different $[\mathrm{Mn} / \mathrm{Fe}]$ trends. 
Acknowledgements. G.C. acknowledges financial support from the Fondazione Cassa di Risparmio di Trieste. We thank P. Bonifacio for his useful suggestions.

\section{References}

Arnett, W. D. 1971, ApJ, 169, 113

Asplund, M., Grevesse, N., \& Sauval, A. J. 2005, ASPC, 336, 25

Badenes, C., Bravo, E., \& Hughes, J. P. 2008, ApJ, 680, L33

Ballero, S. K., Matteucci, F., Origlia, L., \& Rich, R. M. 2007, A\&A, 467, 123

Chiappini, C., Matteucci, F., \& Gratton, R. G. 1997, ApJ, 477, 765

Feltzing, S., \& Gustafsson, B. 1998, A\&AS, 129, 237

François, P., Matteucci, F., Cayrel, R., et al. 2004, A\&A, 421, 613

Gratton, R. G. 1989, A\&A 208, 171

Greggio, L., \& Renzini, A. 1983, MmSAI, 54, 311

Iben, I.Jr., \& Tutukov, A. V. 1984, ApJS, 54,335

Iwamoto, K., Brachwitz, F., Nomoto, K., et al. 1999, ApJS, 125, 439
Lanfranchi, G. A., \& Matteucci, F. 2004, MNRAS, 351, 1338

Lanfranchi, G. A., Matteucci, F., \& Cescutti, G. 2006, MNRAS, 365, 477

Matteucci, F. 2003, Ap\&SS, 284, 539

Matteucci, F., \& Greggio, L. 1986, A\&A, 154, 279

Matteucci, F., \& Recchi, S. 2001, ApJ, 558, 351

McWilliam, A., Rich, R. M., \& Smecker-Hane, T. A. 2003, ApJ, 592, L21

Ohkubo, T., Umeda, H., Nomoto, K., \& Yoshida, T. 2006, AIPC, 847, 458

Reddy, B. E., Tomkin, J., Lambert, D. L., \& Allende Prieto, C. 2003, MNRAS, 340,304

Reddy, B. E., Lambert, D. L., \& Allende Prieto, C. 2006, MNRAS, 367, 1329

Salpeter E. E. 1955, ApJ, 121, 161

Sbordone, L., Bonifacio, P., Buonanno, R., et al. 2007, A\&A, 465, 815

Scalo J. M. 1986, FCPh, 11,

Tinsley, B. M. 1979, ApJ, 229, 1046

Whelan, J., \& Iben, I.Jr. 1973, ApJ, 186, 1007

Woosley, S. E., \& Weaver, T. A. 1995, ApJ, 101, 181 